\title{
Building a business case for digital asset management
}

\author{
Matt Kollmorgen \\ is the VP of Digital Media Solutions for ClearStory Systems. Prior to joining ClearStory, Matt was the Director of \\ Marketing Technologies for Aquent, a global professional services firm, and was responsible for the company's overall \\ technology platform strategy. Matt has also held similar positions at Imation and Kodak. Matt has worked with \\ hundreds of companies across a host of industries on their digital media projects, including the development of ROI \\ models to assess the cost/benefit ratio for technology investments.
}

Keywords: digital asset management (DAM), brand asset management, return on investment (ROI), hosted solutions, total cost of ownership (TCO)

\begin{abstract}
One must look beyond simply managing rich media assets to develop a solid business case. The true return on investment (ROI) for digital asset management (DAM) exists in its effect on speed, efficiency and consistency in all aspects of marketing-related activities for an organization.
\end{abstract}

\section{INTRODUCTION}

As today's enterprises become more sophisticated in the use of digital media - thanks to the introduction of innovative technologies and the need to stand out in a crowded electronic marketplace — the demand for "rich media" is growing exponentially. With that demand comes the need to manage it effectively. As rich media content is distributed globally both within an organization and to external audiences, its value to the organization greatly increases. Traditional print and online channels have already created a large challenge for global organizations to

Matt Kollmorgen Vice President, Digital Media Solutions ClearStory Systems One Research Drive Suite 200B

Westboro, MA 1581 USA

Tel: +1 6175321096 Fax: +1 6173046756 Email: mkollmorgen@ clearstorysystems.com manage valuable content. New channels such as cell phones, PDAs and Podcasting are sure only to add to the complexity - not to mention that these new media types are increasingly expensive to produce and manage. Digital asset management (DAM) solutions enable enterprises to control these costs, maximize the value of rich media assets, and manage the digital rights to this content.

To make the business case for DAM, you need to understand the range of cost factors related to managing and sharing rich media. These often revolve around the time knowledge workers must spend manually performing many of the tasks that can be automated through DAM software. Specifically, such cost factors can include time spent searching for appropriate content, recreating and duplicating rich media files, licensing and rights-management activities, handling requests for file conversions and fulfillment, maintaining archives, and other administrative tasks.

\section{GENERAL ROI BENEFITS OF DAM SOLUTIONS}

DAM can help you achieve cost improvements by enabling greater productivity with more accuracy and 
better controls. Favorable ROI when using such a solution can come from the following areas:

- Cost reduction: The most tangible aspect of savings comes when current business costs are eliminated or reduced, including costs associated with CD or DVD replication, agency fees, shipping costs and file transformation.

- Cost avoidance: Industry analysts estimate that up to 30 per cent of all content is recreated because it is lost or otherwise mismanaged. Avoiding such costs, as well as unnecessary expenses such as stock or custom photo fees, licensing fines and other costs that are a direct result of poorly tracked assets, can contribute to earning returns.

- Strategic value-add resource reallocation: Simply put, your most highly paid workers - knowledge workers - are spending much of their time searching for content. Streamlining searches allows you to reallocate them to focus on more important strategic business objectives.
Many companies fail to convince senior management with the argument that DAM will affect a "hard cost" reduction in search time. In reality, unless DAM will eliminate a dedicated staff position for searching hard drives, CDs and filing cabinets, you still have the fully burdened cost of your knowledge workers. Demonstrating a reallocation of resources to more strategic activities (which can lead to revenue generation see below) is a much more convincing ROI component.

- Revenue generation: Often, a DAM implementation creates new revenue opportunities. For example, a media company may discover that its new library of materials can be repackaged and offered for sale. In nearly all cases, the accelerated delivery of marketing materials means that sales teams, retailers and distributors can respond faster to revenue opportunities. This is a key ROI component for financial services firms looking to deploy DAM. Imagine the ability to arm your field sales agents

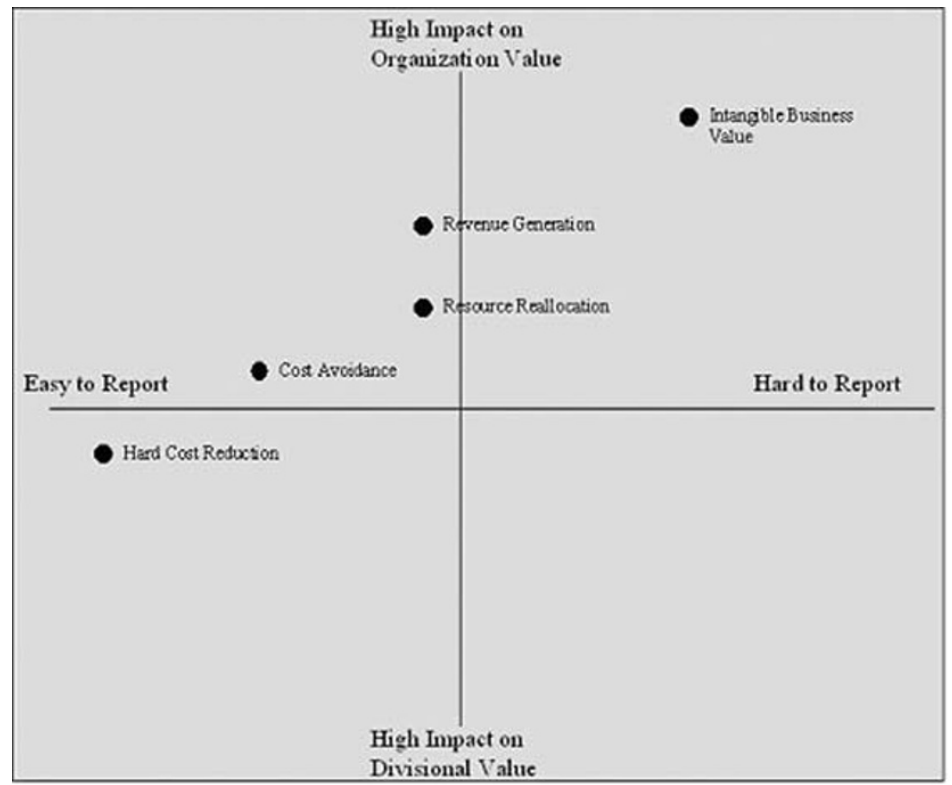

Figure 1: Value that may be hard to quantify typically has the highest return and impact to the organization 
instantly with materials featuring the latest interest rates.

- Intangible business value: This category is the hardest to quantify, yet for some organizations, it is the most critical element (see Figure 1). Better brand management, more consistent messaging, and improving partner relationships are examples of intangible value. Using rich media in a sales presentation may not only save time and money, but also make the presentation more effective and successful. Often, the least tangible benefits come with the greatest benefit to your organization. Organizations that sell through retail channels can easily outsell the competition simply by making it easier for the retail chain to advertise and promote its products or by making feature-benefit information easily available to sales reps.

\section{ACHIEVABLE ROI FOR ALL INDUSTRIES}

It may be counterintuitive, but industries whose core business is the creation and distribution of media do not necessarily see quicker and larger return on investment than other industries. The fact is, any company that builds and launches new brands every day, or manages many different products under one global brand, can achieve the same level of ROI or better by being able to get products to market faster and more efficiently. First-to-market companies can generally enjoy a premium price for their products or services.

Furthermore, there is no simple rule for measuring ROI. Each organization has its own priorities and opportunities for improvement; therefore, ROI data can be found throughout the entire product or service lifecycle and may include all activities from research and development, manufacturing through packaging, marketing and sales. To understand fully how and from which areas the sharing of assets can have a positive effect on ROI, a comprehensive review of each category is needed.

Although hard cost-reduction figures alone can be enough to justify the purchase of a DAM solution, its implementation can also enable more strategic business benefits. For example, one media company justified a DAM purchase to make the management and distribution of video assets more efficient. This exercise presented the company with a strategic revenue opportunity to sell stock footage online. Another company implemented DAM as a global brand portal for sharing marketing materials and collaborating across time zones. This helped them achieve faster time-to-market and overcome some of the cost challenges associated with product launches in new countries.

\section{ROI GROWS AS THE SOLUTION EXPANDS THROUGH THE ENTERPRISE}

Frequently, ROI is calculated for a single business unit for the first DAM deployment. Although different business units may have unique ROI considerations, benefits are also felt between and among interacting units. Widespread adoption of the DAM solution creates a ripple effect in which ROI grows alongside increased utilization.

Consider an organization where the marketing team is able to publish materials more rapidly, and crossdepartmental teams, such as the legal group, can more efficiently review and approve those materials. This is much 
more effective than if the marketing team's time and cost savings are squandered by a long, difficult review process, so the benefit is compounded.

\section{ROI AND TCO FOR IN-HOUSE AND HOSTED SOLUTIONS}

When measuring ROI, a critical consideration is total cost of ownership (TCO). For an in-house solution, the TCO includes the costs of hardware, software, support, professional services, and ongoing administration of the system. If separate business units can utilize the same DAM solution for different applications, TCO is reduced by leveraging the knowledge and/or infrastructure gained through the first deployment. Each additional DAM implementation, therefore, should account for a progressively lower TCO. For an outsourced or hosted DAM solution, while there are no direct equipment costs, you must factor in the ongoing monthly or annual fees.

A significant benefit of a hosted model is faster deployment, which may allow you to take advantage of a market opportunity sooner, enable an accelerated product launch and realize increased revenue as a result. For example, if an internal deployment is delayed 12-18 months due to infrastructure or other concerns, and your ROI model proves returns are possible in six months, it makes sense to outsource and begin earning those returns sooner, even if the upfront costs are slightly higher. For example, if your DAM solution costs US\$300,000, with a six-month projected ROI, the additional US $\$ 300,000-\$ 600,000$ in savings justifies deployment sooner rather than later. If a hosted solution is a preliminary phase to a long-term plan for an installed in-house system, be certain to select a solution that can later be installed in-house.

\section{CONCLUSION}

As you can see, the business case for DAM transcends industry, enterprise adoption rate and the question of inhouse vs outsourced. Hard cost savings, business process efficiency improvements and the ability to take advantage of rapidly emerging market opportunities are contributing factors to ROI and DAM solution justification.

While it may seem like DAM solutions build their own business cases, you should be sure to do your homework and quantify the expected benefits. Understand that as rich media technology and usage evolve, there will be unexpected opportunities to derive additional value from your rich media assets - and, by extension, from the DAM solution itself. 